\title{
Is There a Crystal Lattice Possessing Five-Fold Symmetry?
}

\author{
Michal Kř̌žžek, Jakub Šolc, and Alena Šolcová
}

\section{Kepler's Mosaics}

The German scholar Johannes Kepler (1571-1630), who worked as a court mathematician for the Holy Roman Emperor Rudolf II in Prague, is well known especially for his astronomical discoveries. We still today admire his ability to obtain his three laws of planetary motion from data analysis of Tycho Brahe's observations of Mars. Another outstanding discovery is Kepler's equation describing the connection between the time and the position of a planet on its orbit around the Sun. At present this equation is usually derived from Kepler's first and second laws by means of infinitesimal calculus, but Kepler found a way to obtain it using geometric properties of an ellipse and a circle.

Kepler, however, examined purely mathematical problems as well. Recall, for instance, his famous conjecture on the densest sphere packing. There is still discussion on the validity of its "computer-based proof" given in [12], since it is not in integer arithmetic (only in real computer arithmetic). Kepler is regarded as one of the founders of crystallography. He was astonished by the beauty of snowflakes when he walked over the Charles Bridge in Prague. In his treatise Strena seu de nive sexangula (1611)

Michal Kř́žek is professor of mathematics at the Institute of Mathematics, Academy of Sciences, Czech Republic. His email address is krizek@math. cas.cz.

Jakub Šolc is assistant professor of mathematics at Czech Technical University, Czech Republic. His email address is solc@mat.fsv.cvut.cz.

Alena Šolcová is associate professor of mathematics at Czech Technical University, Czech Republic. Her email address is alena.solcova@fit.cvut.cz.

DOI: http://dx.doi.org/10.1090/noti792. he asked why all snowflakes possess six-fold symmetry even though each one has a different shape. Kepler constructed three interesting star-like polyhedra, for example stella octangula. The other two have six five-fold rotational axes of symmetry; see [23] and Figures 1 and 2.

In his treatise Harmonices mundi (1619) Kepler was dealing with the question of which tilings (i.e., mosaics) of the plane can be made from convex regular $n$-gons such that any two adjacent $n$-gons have an entire edge in common. Moreover, he demanded that each vertex be of the same type $\left(n_{1}, n_{2}, \ldots, n_{k}\right)$, that is, it is surrounded by the regular $n_{1}$-gon, $n_{2}$-gon, and so forth. We shall treat the $k$-tuple $\left(n_{1}, n_{2}, \ldots, n_{k}\right)$ as equivalent to $\left(n_{k}, \ldots, n_{2}, n_{1}\right)$, that is, it is independent of ordering the regular polygons clockwise or counterclockwise. We shall also treat $k$-tuples $\left(n_{1}, n_{2}, \ldots, n_{k}\right)$ and $\left(n_{2}, \ldots, n_{k}, n_{1}\right)$ as equivalent, that is, it does not matter where we start numbering regular polygons. Such a tiling is called semiregular. In particular, if $n_{1}=n_{2}=\cdots=n_{k}$, then it is called regular. Two tilings will be considered equivalent if each can be obtained from the other by translation, rotation, reflection (mirror imaging), dilatation, or composition of such mappings.

Theorem (Kepler). There exist exactly eleven different semiregular tilings of the plane. Three of them are regular.

Let us outline the main idea of the proof (see [15]). The interior angle of the regular $n_{i}$-gon is equal to

$$
\frac{n_{i}-2}{n_{i}} 180^{\circ} .
$$




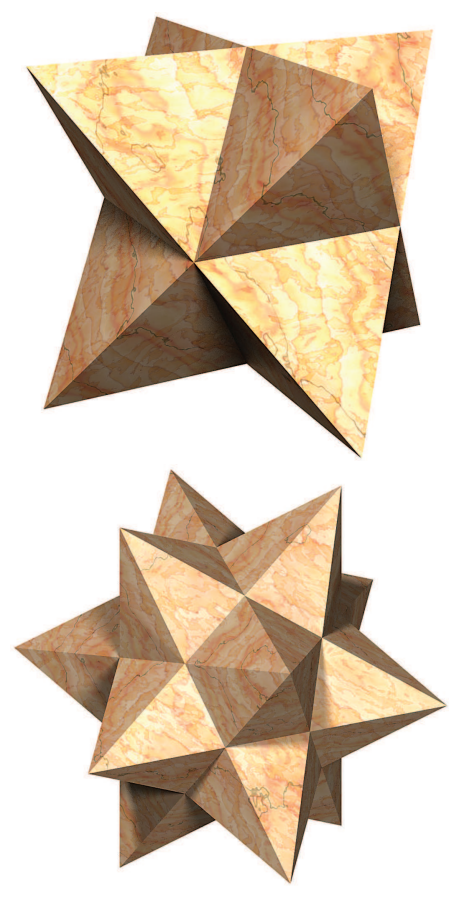

Figure 1. Kepler's star-polyhedra. Stella octangula, top, is the union of two regular tetrahedra. It may be inscribed in a cube. The small stellated dodecahedron is on the bottom. We observe that twelve pentagonal pyramids are, in fact, joined with the twelve pentagonal faces of the regular dodecahedron. Edges of this star-like polyhedron are suitable connections of all vertices of the regular icosahedron.

Therefore, for the vertex of type $\left(n_{1}, n_{2}, \ldots, n_{k}\right)$, the following necessary (but not sufficient) condition for the existence of semiregular tiling holds: $\frac{n_{1}-2}{n_{1}} 180+\frac{n_{2}-2}{n_{2}} 180+\cdots+\frac{n_{k}-2}{n_{k}} 180=360$. From this we come to the Diophantine integer equation

$$
\frac{1}{n_{1}}+\frac{1}{n_{2}}+\cdots+\frac{1}{n_{k}}=\frac{k-2}{2} .
$$

The right-hand side of (1) has to be positive, and thus $k \geq 3$. Since a point can be surrounded by six equilateral triangles and all other $n$-gons have larger inner angles, we obtain the further necessary condition $k \leq 6$ for the solvability of (1). For clarity let us order all entries of the resulting $k$ tuples according to their sizes. Then we get the following seventeen solutions of equation (1). Triples:

$$
\begin{aligned}
& (3,7,42),(3,8,24),(3,9,18),(3,10,15),(3,12,12), \\
& (4,5,20),(4,6,12),(4,8,8),(5,5,10),(6,6,6) ;
\end{aligned}
$$

quadruples:

$$
(3,3,4,12), \quad(3,3,6,6), \quad(3,4,4,6), \quad(4,4,4,4) \text {; }
$$

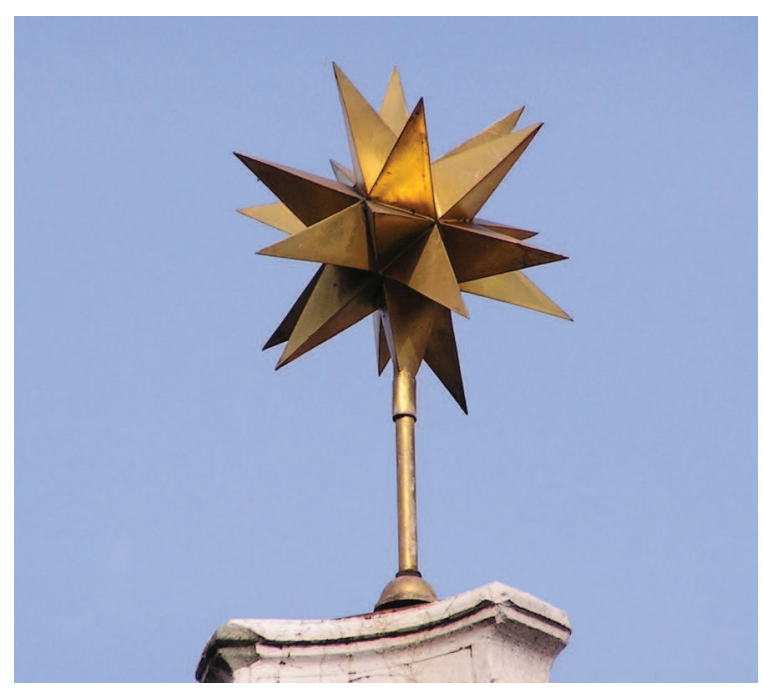

Figure 2. Kepler's great stellated icosahedron in the Charles Square in Prague near the place where Kepler lived. The group of its direct symmetries (i.e., rotation without reflection) is the simple alternating group $A_{5}$, which is used to prove that there does not exist a general formula to evaluate all roots of a polynomial of fifth degree.

quintuples:

$$
(3,3,3,3,6), \quad(3,3,3,4,4) ;
$$

sextuple:

$$
(3,3,3,3,3,3) \text {. }
$$

However, not all of the above solutions correspond to semiregular tilings of the whole plane. For instance, in the case of the solution $(5,5,10)$, we may surround a point by two regular pentagons and one regular decagon, but this is not enough for tiling the whole plane in this way, as one can easily check.

In what follows, we will not require that all entries of the above seventeen $k$-tuples be ordered according to their sizes. Then by inspection of all particular cases we obtain only the following eleven solutions that correspond to tilings of the plane (see Figure 3):

$$
\begin{array}{lll}
(3,3,3,3,3,3), & (4,4,4,4), & (6,6,6), \\
(3,12,12), & (4,8,8), & (4,6,12), \\
(3,6,3,6), & (3,4,6,4), & (3,3,4,3,4), \\
(3,3,3,4,4), & (3,3,3,3,6) . &
\end{array}
$$

Notice that the last two tilings of Figure 3 are mirror images of one another. These two tilings are equivalent, being the left- and right-handed forms of the same tiling. The remaining ten tilings have an axis of symmetry.

Notice that Kepler's semiregular tilings do not have five-fold rotational symmetry, but they have 

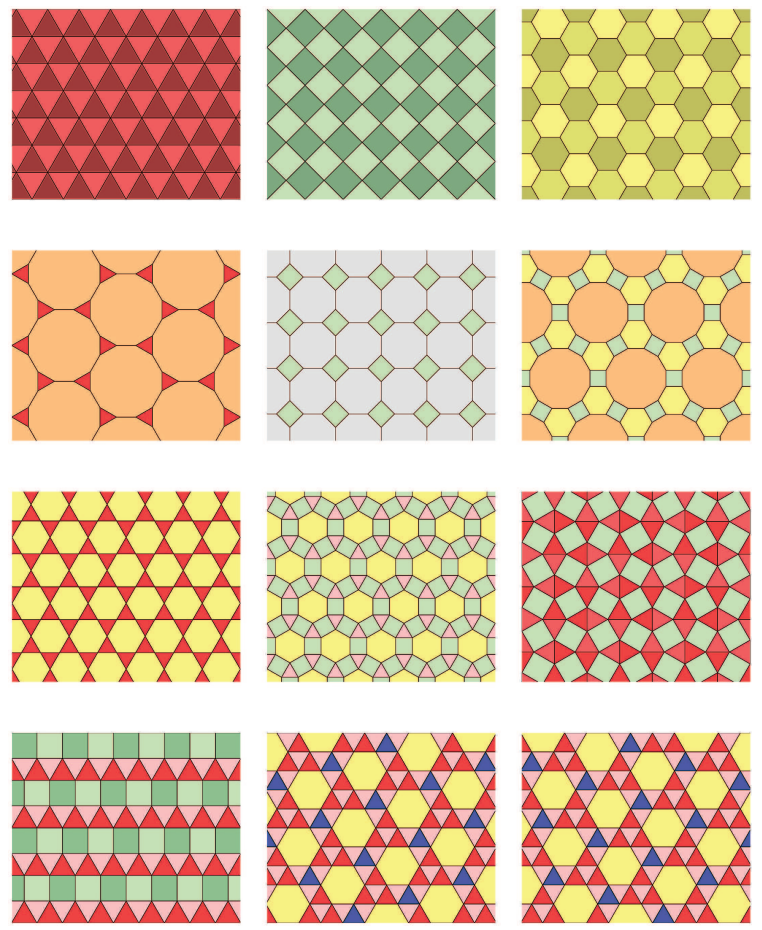

Figure 3. Kepler's mosaics. The last two tilings are mirror images of one another, i. e., they are equivalent. The remaining tilings have an axis of symmetry. Their types are:
$(3,3,3,3,3,3)$,
$(4,4,4,4)$,
$(3,12,12)$,
$(4,8,8)$,
$(6,6,6)$,
$(3,6,3,6)$,
$(3,4,6,4)$,
$(4,6,12)$,
$(3,3,3,4,4)$,
$(3,3,3,3,6)$,
$(3,3,4,3,4)$,
$(3,3,3,3,6)$.

two-, three-, four-, or six-fold symmetry (i.e., rotating each of Kepler's mosaics around an appropriate point about $360^{\circ} / k$ for some $k \in\{2,3,4,6\}$, we get the same mosaic, regardless of its coloring). Anyway, Kepler was dreaming about five-fold symmetry. In his Harmonices mundi we can find several mosaics that have five-point symmetry at least locally (see Figure 4).

In 1850 French mathematician and crystallographer Auguste Bravais proved that there is no five-fold symmetry for three-dimensional crystal lattices.

Note that the whole essay is confined only to Euclidean space and that tiling hyperbolic (or elliptic) space is a completely different problem (see, e.g., some of the famous artworks of M. C. Escher).

\section{Penrose Mosaics}

Let

$$
\alpha=\frac{1+\sqrt{5}}{2} \quad \text { and } \quad \beta=\frac{1-\sqrt{5}}{2} .
$$

They are the roots of the quadratic equation $x^{2}-$ $x-1=0$. The number $\alpha$ is called the golden section (in Latin sectio aurea). It is one of the most
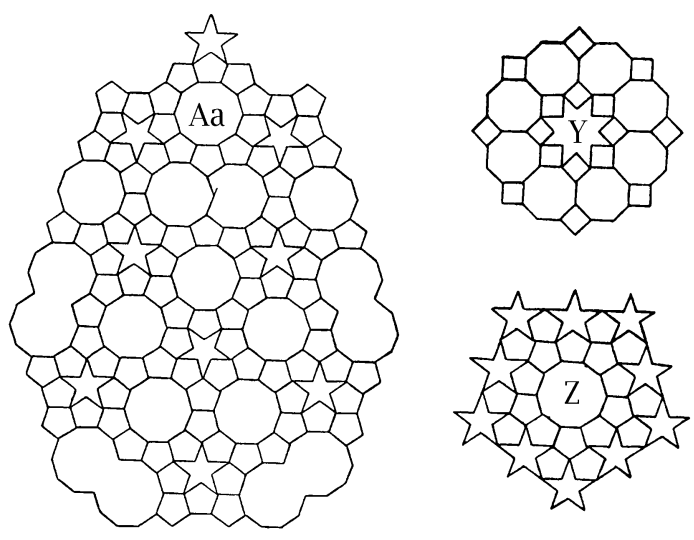

Figure 4. Kepler's experiments with five-fold symmetry in Harmonices mundi. Kepler aligned $n$-gons together to form a cycle. He tried to fill the inner region by other regular $n$-gons, as we may see in figure $Y$ (octagons

and squares). In the same manner he also joined ten pentagons; the inner region forms a regular decagon (Figure $Z$ ). Kepler's major result is shown in Figure $A a$. Note that the overlapping areas of decagons have the exact shape as the smaller Penrose tile.
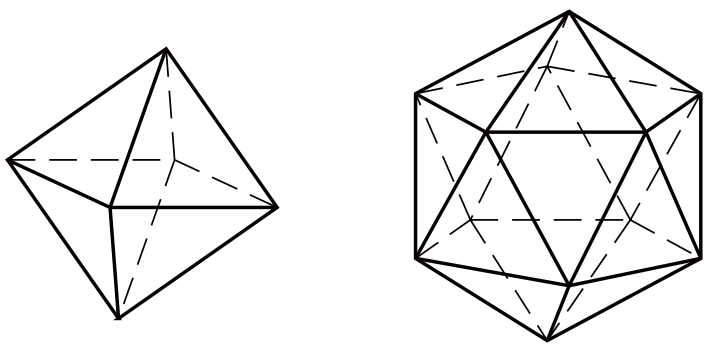

Figure 5. The regular octahedron is on the left. The regular icosahedron on the right has six five-fold rotational axes of symmetry.

mysterious irrational numbers, as we shall see. It appears in some unexpected situations (see, e.g., [13]).

The golden section represents the fundamental aesthetic ratio from antiquity (see [17]). A rectangle whose ratio of edges equals $\alpha$ is generally considered to be the most beautiful. Such a rectangle arises, for instance, as the convex hull of two opposite edges of the regular icosahedron (see Figure 5). The ratio of lengths of the diagonal and an edge of the regular pentagon is $\alpha$. Also the length of an edge of the regular pentagon is the larger part of its diagonal when it is divided into two line segments whose lengths have ratio $\alpha$. The ratio of the radius of the circumscribed circle to the regular decagon and the length of its edge is also $\alpha$. The volume of the regular dodecahedron and icosahedron is $2+7 \alpha / 2$ and $5(1+\alpha) / 6$, 
respectively, provided the length of their edges is equal to 1 .

Johannes Kepler discovered a polyhedron with thirty congruent faces (the so-called Kepler's rhombic triacontahedron) which is the intersection of five differently oriented cubes with the same center (cf. Figures 6 and 7). All thirty faces have rhombic shape, and the ratio of their diagonals is $\alpha=-1 / \beta$, too. The same faces are found in the rhombic hexecontahedron which was discovered by $\mathrm{H}$. Unkelbach around 1940 (see [9], [27]). Amazingly, this sixty-faced solid (which was chosen as the logo of WolframAlpha) is believed to exist in nature as the central core of a quasicrystal aggregate of $\mathrm{Al}_{6} \mathrm{Li}_{3} \mathrm{Cu}$ produced by slow solidification (see [11]).

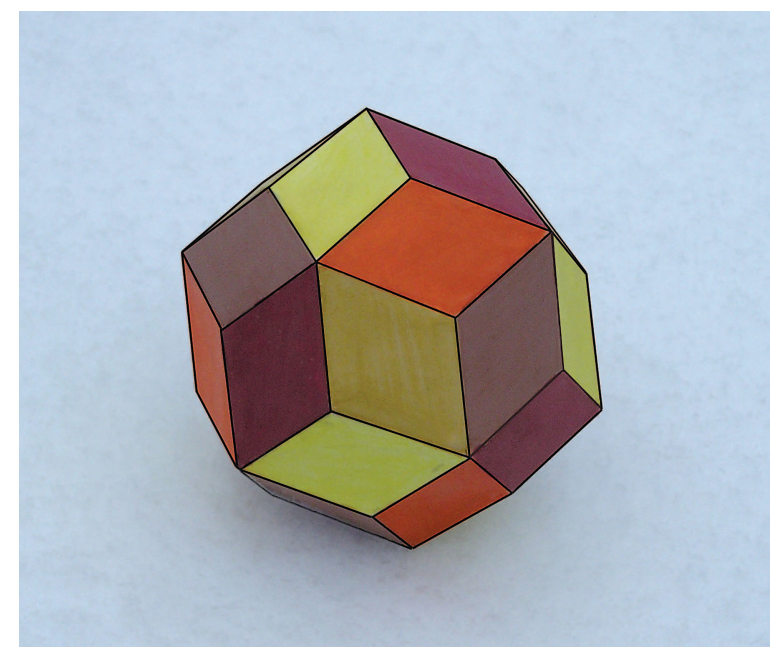

Figure 6. Kepler's rhombic triacontahedron is the intersection of five differently oriented and differently colored cubes with the same center. This polyhedron has six five-fold rotational axes of symmetry.

The coordinates of vertices of the regular fourdimensional simplex with center at the origin can also be expressed by means of the golden section, namely

(3)

$$
\begin{array}{ll}
v_{1}=(3 \alpha-1, \beta, \beta, \beta)^{\top}, & v_{2}=(\beta, 3 \alpha-1, \beta, \beta)^{\top}, \\
v_{3}=(\beta, \beta, 3 \alpha-1, \beta)^{\top}, & v_{4}=(\beta, \beta, \beta, 3 \alpha-1)^{\top}, \\
v_{5}=(-2,-2,-2,-2)^{\top} . &
\end{array}
$$

In four-dimensional space there exists another regular polytope-the 600 cell, which is really an extraordinary mathematical object. Its threedimensional boundary consists of 600 regular tetrahedra (see, e.g., [6], [25]). This hypersurface contains 720 edges that form, altogether, 72 edge-disjoint regular decagons with a common center. (Similarly, the surface of the regular octahedron contains twelve edges that form three

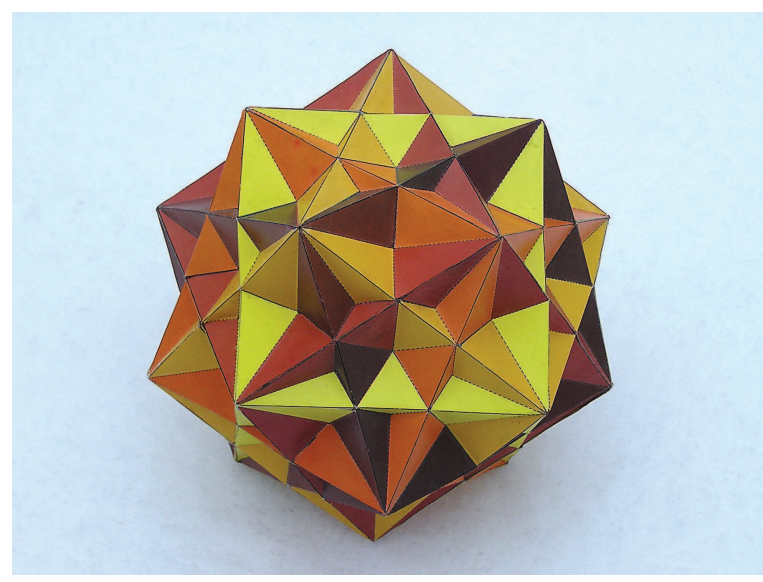

Figure 7. The union of the five cubes whose intersection is Kepler's polyhedron. It also has six five-fold rotational axes of symmetry, and its convex hull is the regular dodecahedron (and, conversely, the convex hull of eight suitably chosen vertices of the dodecahedron is a cube).

edge-disjoint squares.) The ratio of the radius of the circumscribed ball to the 600 cell and the length of its edge is again $\alpha$. There are other analogous examples.

In the beginning of the 1970s the British mathematician and physicist Roger Penrose discovered tilings of the plane by two types of rhombic tiles that possess a local five-fold symmetry (see Figure 8 ) and have a close connection to the golden section. The edges of both tiles have the same length. The first tile has an acute angle equal to $72^{\circ}$ and

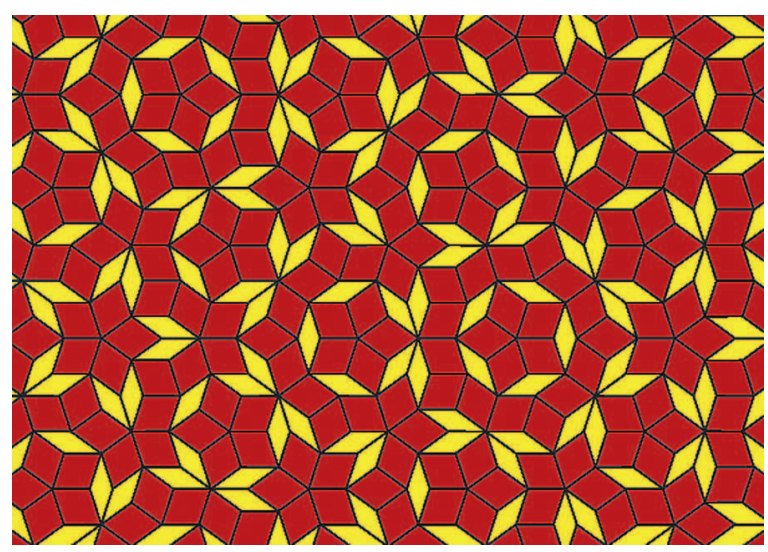

Figure 8. Penrose nonperiodic tiling.

the second equal to $36^{\circ}$. They are called Penrose tiles.

We can easily verify that

$$
\cos 72^{\circ}=\frac{1}{2 \alpha} \text {. }
$$


Assume that the length of the edges of both tiles is equal to 1 . Then the area of the first Penrose tile is $\sin 72^{\circ}=2 \sin 36^{\circ} \cos 36^{\circ}$ and $\sin 36^{\circ}$ of the second tile. Thus the ratio of their areas is again the golden section

$$
\sin 72^{\circ}: \sin 36^{\circ}=2 \cos 36^{\circ}=\alpha .
$$

Penrose himself admits that he was inspired by Kepler's work Harmonices mundi, in which tiling by pentagonal tiles is examined as well. To construct the Penrose tiling it is necessary that all tiles satisfy the so-called Penrose rule:

It is necessary to join tiles so that the bold curves of the same color meet (see Figure 9).
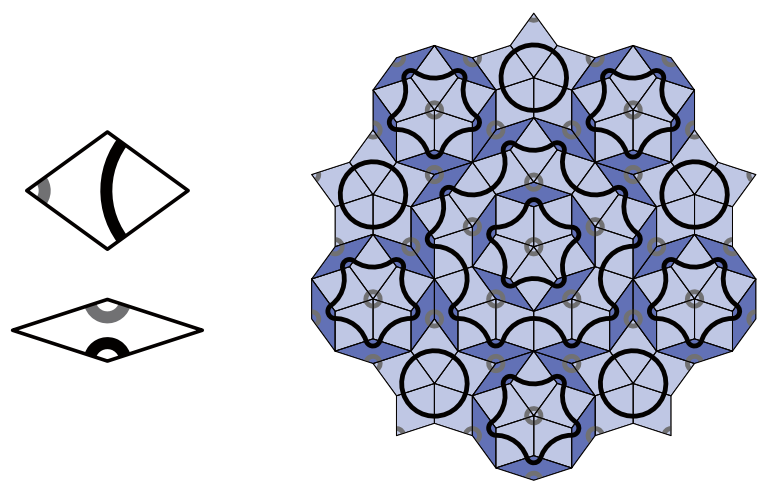

Figure 9. To generate a nonperiodic Penrose tiling it is necessary to join Penrose tiles so that the bold curves of the same color meet.

Then we may even get a mosaic that possesses the five-fold symmetry globally with respect to one point.

The most surprising property of Penrose tilings is that they do not admit a nontrivial translative symmetry (see Figure 8). Shifting the Penrose tiling about any vector will never be consistent with the original tiling, even though the possible number of combinations of tiles surrounding a given vertex is finite. The following definition is from [22]: A tiling is said to be nonperiodic if it does not admit any nontrivial transitive symmetry.

There is a large amount of literature on Penrose tilings. We mention here only the original article [20], in which the rhombic tiles from Figure 9 appear for the first time.

Theorem (Penrose). Every tiling of the plane by Penrose tiles that satisfies the Penrose rule is nonperiodic.

For the proof we refer to the monograph [10, Chapter 10].

When the Penrose rule is violated, we can form periodic tilings from the Penrose tiles. For instance, we may arrange these tiles into infinite strips, which may then be used to construct periodic tilings. For construction of a nonperiodic tiling it is not enough to assume that any two adjacent tiles do not form a parallelogram. We may generate periodic tiling from Penrose tiles, for example, so that each vertex is surrounded by four tiles with angles $36^{\circ}, 72^{\circ}, 144^{\circ}$, and $108^{\circ}$ (always in this order). In this case the Penrose rule is also not satisfied. Penrose tilings can be generalized into higher-dimensional spaces.

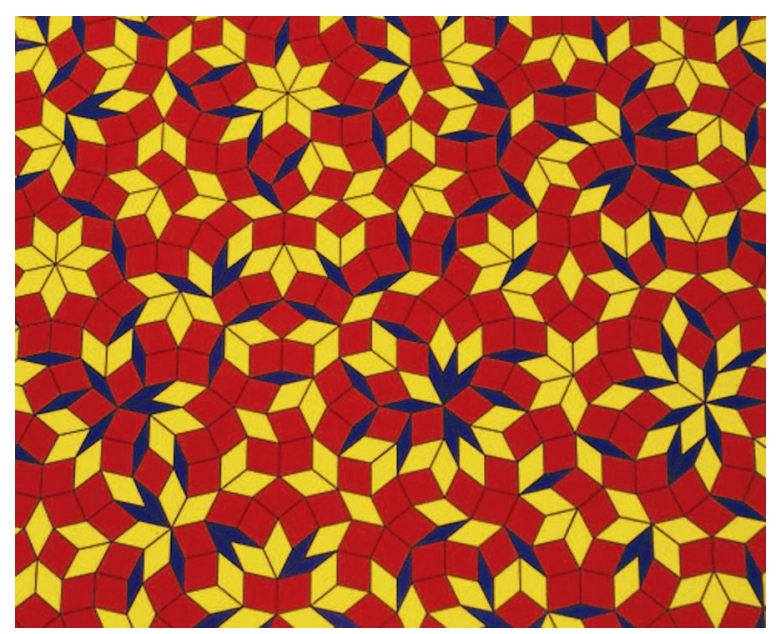

Figure 10. Katz nonperiodic tiling.

In 1986 André Katz found nonperiodic tilings of the plane that possess a local seven-fold rotational symmetry (see Figure 10). His three types of tiles again have rhombic shapes, whose acute angles are successively $180^{\circ} / 7,360^{\circ} / 7$, and $540^{\circ} / 7$. For more details see [21], [26].

Note that the plane can be tiled nonperiodically using tiles of a single shape, for instance, by right isosceles triangles. It is enough to consider the famous Ulam spiral (see Figure 11), which was proposed in 1963 by the Polish mathematician Stanislaw Marcin Ulam (1909-1984). Prime numbers are written in black squares and the others in white squares. All black squares will be divided by the diagonals with slope 1 into two right isosceles triangles. The other squares will be divided by the diagonals with slope $(-1)$. This gives the required nonperiodic tiling with one tile only.

\section{Semiregular Polyhedra}

In his dialogue treatise Timaios the Greek philosopher Plato (427-347 B.C.) mentioned five regular polytopes in the three-dimensional space. Recall that a regular polyhedron (also Platonic solid) is a convex polyhedron whose faces are congruent regular polygons and in which each vertex is surrounded by the same number of faces. Note that the assumption of convexity is essential. There exists a nonconvex polyhedron whose surface consists of twenty congruent equilateral triangles in which each vertex is surrounded by five faces. 


\begin{tabular}{|c|c|c|c|c|c|c|c|c|c|}
\hline 91 & 90 & 89 & 88 & 87 & 86 & 85 & 84 & 83 & 82 \\
\hline 92 & 57 & 56 & 55 & 54 & 53 & 52 & 51 & 50 & 81 \\
\hline 93 & 58 & 31 & 30 & 29 & 28 & 27 & 26 & 49 & 80 \\
\hline 94 & 59 & 32 & 13 & 12 & 11 & 10 & 25 & 48 & 79 \\
\hline 95 & 60 & 33 & 14 & 3 & 2 & 9 & 24 & 47 & 78 \\
\hline 96 & 61 & 34 & 15 & 4 & 1 & 8 & 23 & 46 & 77 \\
\hline 97 & 62 & 35 & 16 & 5 & 6 & 7 & 22 & 45 & 76 \\
\hline 98 & 63 & 36 & 17 & 18 & 19 & 20 & 21 & 44 & 75 \\
\hline 99 & 64 & 37 & 38 & 39 & 40 & 41 & 42 & 43 & 74 \\
\hline 100 & 65 & 66 & 67 & 68 & 69 & 70 & 71 & 72 & 73 \\
\hline
\end{tabular}

Figure 11. Ulam's square spiral of positive integers. The background color of prime numbers is black.

A semiregular polyhedron is a convex polyhedron whose faces are regular polygons and whose spatial angles at vertices are directly or indirectly congruent. Note that a spatial angle is indirectly congruent with its mirror image.

Platonic solids are special cases of semiregular polyhedra. Their boundary is formed by polygons of a single shape. Semiregular polyhedra whose surfaces are formed by two or more types of polygons are classified into Archimedean solids, regular prisms, and regular antiprisms. Their existence can be investigated similarly as semiregular tilings of the plane by means of Diophantine inequalities as in the proof of Kepler's theorem. The regular prisms (resp., regular antiprisms) have two opposite faces formed by a regular $n$-gon, and the other faces are squares (resp., equilateral triangles). A special case of the regular prism (resp., regular antiprism) is the cube (resp., regular octahedron). A survey of the thirteen Archimedean solids is given in the second chapter of Harmonices mundi by Johannes Kepler.

Around 1905 a fourteenth Archimedean solid was discovered that can be obtained by rotating the "upper layer" of the tenth body from Figure 12 about $45^{\circ}$ (see [24, Figure 27]). It has no point of symmetry. The last two polyhedra in Figure 12 exist in left- and right-handed forms, like Kepler's last two tilings in Figure 3. Terminology (what is meant by an Archimedean solid) is unfortunately not unique. Notice that there are several semiregular polyhedra that have five-fold rotational symmetry with respect to six axes.

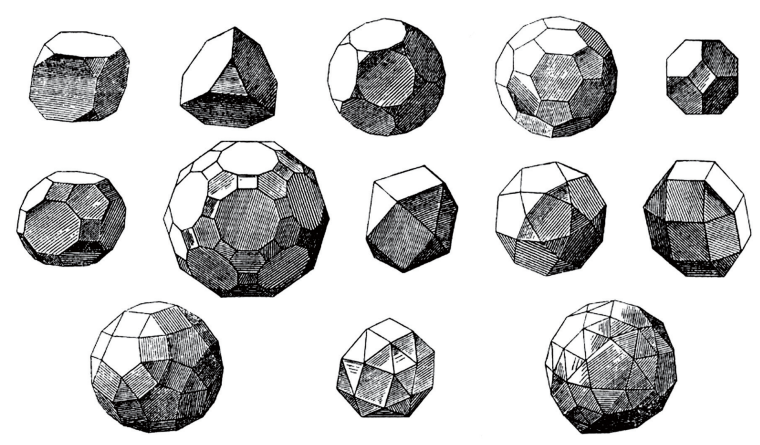

Figure 12. Archimedean solids from Kepler's treatise Harmonices mundi.

The classic football (soccer ball) resembles an Archimedean solid with twelve pentagonal and twenty hexagonal faces (see the fourth body in Figure 12). This polyhedron has sixty vertices and appears as a model in chemistry, since there exists a stable carbon molecule, the so-called fullerene $\mathrm{C}_{60}$, that has sixty atoms placed at vertices of such a semiregular polyhedron. In addition, pollen dust and viruses sometimes have the shape of semiregular polyhedra.

Construction of a partition of a ball surface is often derived from partitions of triangular faces of the regular icosahedron. The regular icosahedron (see Figure 5) has been used to partition three-dimensional space into acute tetrahedra (see [8] and [2]). The cube is the only Platonic solid that can be used to tile three-dimensional space. Three-dimensional space can also be tiled by regular tetrahedra and regular octahedra arranged in such a way that each triangular face is common just to one tetrahedron and one octahedron. A diamond has such a crystal lattice, and therefore it is the hardest of all minerals.

Let us point out that Archimedean solids can be generalized to higher-dimensional spaces $\mathbb{R}^{d}$. For $d>3$ there exist exactly seven semiregular polytopes (except for regular polytopes, prisms, and antiprisms); three of them lie in dimension $d=4$ and one each in dimensions $d=5,6,7,8$ (see [1]). For instance, in four-dimensional space there exists a semiregular polytope that has ten vertices and whose surface is formed by five regular tetrahedra and five regular octahedra. It possesses fivefold symmetry.

The space $\mathbb{R}^{8}$ can be tiled by a semiregular polytope whose group of symmetry is $E_{8}$ (see [28]). It is worth mentioning that inscribed balls to these bodies form the densest sphere packing in $\mathbb{R}^{8}$ (see [16] and [19]). Each ball touches 240 other balls. This property is applied in the construction of one of the most effective Hamming error-correcting 
codes. The set of centers of these balls is

$\left\{\left(x_{1}, x_{2}, \ldots, x_{8}\right) \in \mathbb{Z}^{8} \cup\left(\mathbb{Z}+\frac{1}{3}\right)^{8} \mid \sum_{i=1}^{8} x_{i} \equiv 0 \bmod 2\right\}$.

Voronoi cells corresponding to these centers form just particular copies of this interesting semiregular body.

\section{Five-Fold Symmetry in Four-Dimensional Space}

A crystal lattice is formed by the edges of mutually congruent parallelepiped cells defined by three linearly independent vectors $a_{1}, a_{2}, a_{3} \in \mathbb{R}^{3}$ (see Figure 13). We shall assume that the lattice is unbounded and that it has a translation symmetry with respect to integer multiples of the vectors $a_{i}$. The French crystallographer Auguste Bravais (1811-1863) investigated point groups of symmetries of crystal lattices in $\mathbb{R}^{3}$, that is, isometric mappings of the whole lattice onto itself, which have at least one fixed point (see [4]). Here symmetries with respect to direct congruent transformations are meant (i.e., reflections and rotatory reflections are not considered). Symmetries of such lattices can be rotations about an axis through the angle $k \cdot 360^{\circ} / n$, where $k$ is an integer and $n \in\{1,2,3,4,6\}$. Crystal lattices thus may have two-, three-, four-, and six-fold symmetry but not five-fold symmetry. As an immediate consequence we get the following theorem.

Theorem (Bravais). There is no crystal lattice in $\mathbb{R}^{3}$ with five-fold symmetry.

The five-fold symmetry may appear only locally, for instance in quasicrystals whose atoms are arranged into regular icosahedra that are separated by gaps (see [18]). By means of Coxeter groups Chen, Moody, and Patera [5] give a detailed analysis of noncrystallographic root system based on five-fold symmetry and the quasicrystals that are associated with them.

Consider now an unbounded crystal lattice in higher dimensions defined by linearly independent vectors $a_{1}, a_{2}, \ldots, a_{d} \in \mathbb{R}^{d}$. Denote by

$$
V=\left\{\sum_{i=1}^{d} c_{i} a_{i} \mid c_{i} \in \mathbb{Z}\right\}
$$

its set of vertices. Recall that a mapping $\mathcal{A}: V \rightarrow V$ is said to be an isometry if it preserves distances, that is, $\|\mathcal{A}(x)\|=\|x\|$ for all $x \in V$, where $\|\cdot\|$ is the Euclidean norm.

We say that the lattice has a five-fold symmetry if there exists a nonidentical isometry mapping $\mathcal{A}: V \rightarrow V$ such that

$$
\mathcal{A}^{5}(x)=x \quad \text { for all } x \in V .
$$

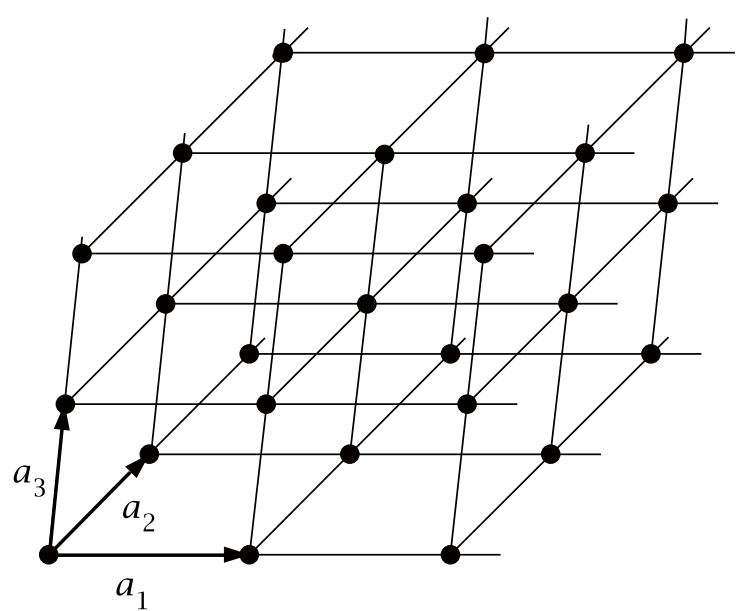

Figure 13. Crystal lattice.

Let $e_{i}=(0,0, \ldots, 0,1,0, \ldots, 0)$ be the standard basis vector with $d$ entries having 1 at the $i$ th position. Let

$$
P=\left[\begin{array}{ccccc}
0 & 0 & \ldots & 0 & 1 \\
1 & 0 & \ldots & 0 & 0 \\
0 & 1 & \ldots & 0 & 0 \\
& & \ddots & & \\
0 & 0 & \ldots & 1 & 0
\end{array}\right]
$$

be a $d \times d$ permutation matrix. Then we get

$$
e_{i+1}=P e_{i}, \quad i=1, \ldots, d-1, \quad \text { and } \quad e_{1}=P e_{d} .
$$

Hence we arrive at:

Corollary. For any integer $d \geq 2$ the power $P^{d}$ is the unit $d \times d$ matrix.

As an immediate consequence we find that the hypercube lattice in $\mathbb{R}^{5}$ has a five-fold symmetry. Thus a natural question arises: Does there exist $a$ lattice with five-fold symmetry in spaces of dimension less than five?

The answer is positive for $d=4$ (see, e.g., [7], [18]). However, it is not easy to visualize a complicated geometry in four dimensions. For $\alpha$ and $\beta$ from relations (2) we define linearly independent unit vectors

$$
\begin{array}{ll}
a_{1}=(1,0,0,0)^{\top}, & a_{2}=\left(-\frac{1}{2}, \frac{\alpha}{2}, \frac{\beta}{2}, 0\right)^{\top}, \\
a_{3}=\left(0,-\frac{\beta}{2},-\frac{\alpha}{2}, \frac{1}{2}\right)^{\top}, & a_{4}=(0,0,0,-1)^{\top}
\end{array}
$$

and mirror image mappings $r_{i}$ by (see [18]):

$$
\begin{aligned}
& r_{i}\left(a_{i}\right)=-a_{i}, \\
& r_{i}\left(a_{j}\right)=a_{i}+a_{j} \quad \text { for }|i-j|=1, \\
& r_{i}\left(a_{j}\right)=a_{j} \quad \text { for }|i-j|>1,
\end{aligned}
$$

where $i, j \in\{1,2,3,4\}$. Later (see (6) below) we show that the $r_{i}$ are uniquely determined. From (4) we can easily verify that the sum of two (or more) vectors $a_{i}$ with adjacent indices is again a unit vector. 
The mirror plane of $r_{i}$ is orthogonal to the vector $a_{i}$ and passes through the origin. The mirror planes corresponding to $r_{i}$ and $r_{j}$ with adjacent indices form an angle of $60^{\circ}$, since $a_{i}^{\top} a_{j}= \pm \frac{1}{2}$. If the difference of the indices $i$ and $j$ in absolute value is larger than one, then the mirror planes of $r_{i}$ and $r_{j}$ are orthogonal.

The set of vertices of the corresponding crystal lattice in $\mathbb{R}^{4}$,

$$
V=\left\{\sum_{i=1}^{4} c_{i} a_{i} \mid c_{i} \in \mathbb{Z}\right\},
$$

clearly has a translation symmetry in integer multiples of each direction $a_{i}$. The lattice itself consists of edges of congruent four-dimensional parallelepiped cells. Now we prove that it has a fivefold symmetry for $d=4$.

Theorem. There exists a nonidentical isometry $\mathcal{A}$ from the set $V$ into itself such that

$$
\mathcal{A}^{5}(x)=x \quad \forall x \in V .
$$

Proof. Define the isometry mapping

$$
\mathcal{A}=r_{1} r_{3} r_{2} r_{4} .
$$

Then by (5) we get

$$
\begin{aligned}
\mathcal{A}\left(a_{1}\right) & =r_{1} r_{3} r_{2}\left(a_{1}\right)=r_{1} r_{3}\left(a_{1}+a_{2}\right) \\
& =r_{1}\left(a_{1}+a_{2}+a_{3}\right)=a_{2}+a_{3}, \\
\mathcal{A}\left(a_{2}\right) & =r_{1} r_{3} r_{2}\left(a_{2}\right)=-r_{1} r_{3}\left(a_{2}\right) \\
& =-r_{1}\left(a_{2}+a_{3}\right)=-a_{1}-a_{2}-a_{3}, \\
\mathcal{A}\left(a_{3}\right) & =r_{1} r_{3} r_{2}\left(a_{3}+a_{4}\right)=r_{1} r_{3}\left(a_{2}+a_{3}+a_{4}\right) \\
& =r_{1}\left(a_{2}+a_{3}+a_{4}\right)=a_{1}+a_{2}+a_{3}+a_{4}, \\
\mathcal{A}\left(a_{4}\right) & =-r_{1} r_{3} r_{2}\left(a_{4}\right)=-r_{1} r_{3}\left(a_{4}\right) \\
& =-r_{1}\left(a_{3}+a_{4}\right)=-a_{3}-a_{4} .
\end{aligned}
$$

From this we find that $\mathcal{A}$ is a linear operator from the discrete set $V$ into $V$ which maps the following elements as:

$$
\begin{aligned}
a_{1} & \mapsto a_{2}+a_{3} \mapsto a_{4} \mapsto-\left(a_{3}+a_{4}\right) \mapsto-\left(a_{1}+a_{2}\right) \mapsto a_{1}, \\
a_{2} & \mapsto-\left(a_{1}+a_{2}+a_{3}\right) \mapsto-\left(a_{2}+a_{3}+a_{4}\right) \\
& \mapsto a_{3} \mapsto a_{1}+a_{2}+a_{3}+a_{4} \mapsto a_{2} .
\end{aligned}
$$

We observe that $\mathcal{A}^{5}\left(a_{i}\right)=a_{i}$ for all $i=1,2,3,4$, and thus $\mathcal{A}^{5}$ is the identity.

Let us take a closer look at the operator $\mathcal{A}$ from the previous theorem. We will express it by means of matrix theory. The mappings $r_{i}$ are uniquely defined by the orthogonal matrices of mirror imaging

$$
A_{i}=I-2 a_{i} a_{i}^{\top}, \quad i=1,2,3,4,
$$

i.e., $r_{i}(x)=A_{i} x$ for $x \in \mathbb{R}^{4}$, where

$$
A_{1}=\left[\begin{array}{cccc}
-1 & 0 & 0 & 0 \\
0 & 1 & 0 & 0 \\
0 & 0 & 1 & 0 \\
0 & 0 & 0 & 1
\end{array}\right], \quad A_{2}=\frac{1}{2}\left[\begin{array}{llll}
1 & \alpha & \beta & 0 \\
\alpha & \beta & 1 & 0 \\
\beta & 1 & \alpha & 0 \\
0 & 0 & 0 & 2
\end{array}\right],
$$

$A_{3}=\frac{1}{2}\left[\begin{array}{llll}2 & 0 & 0 & 0 \\ 0 & \alpha & 1 & \beta \\ 0 & 1 & \beta & \alpha \\ 0 & \beta & \alpha & 1\end{array}\right], \quad A_{4}=\left[\begin{array}{cccc}1 & 0 & 0 & 0 \\ 0 & 1 & 0 & 0 \\ 0 & 0 & 1 & 0 \\ 0 & 0 & 0 & -1\end{array}\right]$.

Then $\mathcal{A}$ is given by the orthogonal matrix

$$
A=A_{1} A_{3} A_{2} A_{4}=\frac{1}{2}\left[\begin{array}{cccc}
-1 & -\alpha & -\beta & 0 \\
1 & 0 & \alpha & -\beta \\
1 & \beta & 0 & -\alpha \\
-1 & 1 & 1 & -1
\end{array}\right] .
$$

For $\varphi=72^{\circ}$ and $\psi=144^{\circ}$ the eigenvalues of $A$ are

(7) $\lambda_{1,2}=\cos \varphi \pm i \sin \varphi, \quad \lambda_{3,4}=-\cos \psi \pm i \sin \psi$.

Since they are not real, the only fixed point of the mapping $\mathcal{A}(x)=A x$ is zero. According to the previous theorem, $A^{5}$ is the identity matrix. Let us still note that the eigenvalues of the matrix

$$
A^{4}=A^{-1}=A^{\top}=\frac{1}{2}\left[\begin{array}{cccc}
-1 & 1 & 1 & -1 \\
-\alpha & 0 & \beta & 1 \\
-\beta & \alpha & 0 & 1 \\
0 & -\beta & -\alpha & -1
\end{array}\right]
$$

are the same as those of the matrix $A$.

Remark. The operator $\mathcal{A}$ from the previous theorem and the corresponding matrix $A$ are, of course, not unique. Setting, for instance,

$$
B=\left[\begin{array}{llll}
a & b & c & c \\
a & c & b & c \\
a & c & c & b \\
d & a & a & a
\end{array}\right],
$$

where $a=-\alpha / 4, b=(\alpha+2) / 4, c=-(\beta+1) / 4$, and $d=(3 \beta-1) / 4$, we find that $B^{5}$ is also the identity matrix. This follows from the fact that

(8) $v_{i+1}=B v_{i}, \quad i=1,2,3,4$, and $v_{1}=B v_{5}$, where $v_{i}$ are vertices of the regular four-dimensional simplex (see (3)). Hence $B$ is the orthogonal matrix, since $B^{4}=B^{-1}=B^{\top}$. The eigenvalues of $B$ are the same as those in (7). Another block diagonal matrix (cf. again (7))

$$
D=\left[\begin{array}{cccc}
\cos \varphi & -\sin \varphi & 0 & 0 \\
\sin \varphi & \cos \varphi & 0 & 0 \\
0 & 0 & \cos \varphi & -\sin \varphi \\
0 & 0 & \sin \varphi & \cos \varphi
\end{array}\right]
$$

with similar properties is given in [7, p. 80].

Analogously we can prove that there exists a lattice with seven-fold symmetry in $\mathbb{R}^{6}$. It is enough to consider the regular six-dimensional simplex and relations similar to (8).

\section{Conclusions}

At the beginning of the seventeenth century, Johannes Kepler was thinking about periodic mosaics with five-fold symmetry. Also many other scholars wanted to discover planar mosaics or spatial crystal lattices with such a symmetry. However, in 1850 Auguste Bravais proved that crystal 
lattices in two- and three-dimensional space may have only two-fold, three-fold, four-fold, or six-fold symmetry.

In this paper we showed that five-fold symmetry appears in special crystal lattices in four-dimensional space, and we gave an explicit algorithm to construct it. Five-fold symmetry in lower-dimensional Euclidean spaces can appear only locally, which can be seen, for example, in paintings of some of Kepler's mosaics or in the famous Penrose tilings in the early 1970s. Projections from physically abstract more-dimensional constructs produce real space maps with local symmetries that show correlations with the quasicrystal atomic arrangements in some alloys, which may have many applications in the future.

\section{Acknowledgment}

This research was supported by Grant No. IAA 100190803 of the Academy of Sciences of the Czech Republic and Institutional Research Plan AV0Z 10190503. The authors are thankful to Jan Brandts (University of Amsterdam) for fruitful discussions.

\section{References}

[1] G. BLIND and R. BLIND, The semiregular polytopes, Comment. Math. Helvetici 66 (1991), 150-154.

[2] J. BRAndTS, S. Korotov, M. KřížEK, and J. ŠolC, On nonobtuse simplicial partitions, SIAM Rev. 51 (2009), 317-335.

[3] S. BARANidhaRAN, V. S. K. Balagurusamy, A. SRINIVASAN, E. S. R. GOPAL, and V. SASISEKHARAN, Nonperiodic tilings in 2-dimensions: 4- and 7-fold symmetries, Phase Transition 16 (1989), 621-626.

[4] A. BRAvais, Mémoire sur les systèmes formés par les points distribués régulièrement sur un plan ou dans l'espace, J. Ecole Polytech. 19 (1850), 1-128.

[5] L. Chen, R. V. Moody, and J. PATERA, Noncrystallographic root systems, in Quasicrystals and Discrete Geometry (J. Patera, ed.), Fields Inst. Monographs, vol. 10, 1998, 135-178.

[6] H. S. M. CoXeter, Regular Polytopes, Methuen, London, New York, 1948, 1963.

[7] P. ENGEL, Geometric Crystallography: An Axiomatic Introduction to Crystallography, D. Reidel, Boston, Lancaster, Tokyo, 1986.

[8] D. Eppstein, J. M. Sullivan, and A. ÜngöR, Tiling space and slabs with acute tetrahedra, Comput. Geom.: Theory and Appl. 27 (2004), 237-255.

[9] B. GRÜNBAUM, Dodecahedron and assorted parallelohedra, zonohedra, monohedra, isozonohedra and otherhedra, Math. Intelligencer 32 (2010), no. $4,5-15$.

[10] B. GrünbAum and G. C. ShePhARD, Tilings and Patterns, W. H. Freeman, New York, 1987.

[11] P. GuYOT, News on five-fold symmetry, Nature 326 (1987), 640-641.

[12] T. C. HALES, Cannonballs and honeycombs, Notices Amer. Math. Soc. 47 (2000), 440-449.

[13] T. Koshy, Fibonacci and Lucas Numbers with Applications, Wiley, New York, 2001.
[14] F. Krafft (ed.), Johannes Kepler: Was die Welt im Innersten zusammenhält-Antworten aus Keplers Schriften mit einer Enleitung, Erläuterungen und Glossar herausgegeben von Fritz Krafft, Marix Verlag, GmbH, Wiesbaden, 2005.

[15] M. KŘížEK and J. Šolc, From Kepler's mosaics to five-fold symmetry (in Czech), Pokroky Mat. Fyz. Astronom. 54 (2009), 41-56.

[16] V. I. LEVENSHTEIN, On bounds for packing in $n$ dimensional Euclidean space, Soviet Math. Dokl. 20 (1979), 417-421.

[17] M. LIVIO, The Golden Ratio, Headline, London, 2002.

[18] Z. MASÁkovÁ, J. PATERA, and E. PELANTovÁ, Inflation centers of the cut and project quasicrystal, $J$. Phys. A: Math. Gen. 31 (1998), 1443-1453.

[19] A. M. ODLYZKo and N. J. A. SLOANE, New bounds on the number of unit spheres that can touch a unit sphere in $n$ dimensions, J. Combin. Theory Ser. A 26 (1979), 210-214.

[20] R. PENROSE, Role of aesthetics in pure and applied mathematical research, Bull. Inst. Maths. Appl. 10 (1974), 266-271.

[21] V. SASISEKHARAN, A new method for generation of quasi-periodic structures with $n$ fold axes: Application to five and seven folds, Pramana 26 (1986), 283-293.

[22] E. Schulte, Tilings, in Encyclopedia of Physical Science and Technology, third ed., vol. 16, Academic Press, San Diego, 2001, 273-282.

[23] A. Šolcová, Johannes Kepler, The Founder of Celestial Mechanics (in Czech), Prometheus, Prague, 2004.

[24] D. M. Y. SommeRville, Semi-regular networks of the plane in absolute geometry, Trans. Roy. Soc. Edinburgh 41 (1905), 725-747.

[25] J. STILlWELl, The story of the 120-cell, Notices Amer. Math. Soc. 48 (2001), 17-24.

[26] A. SubramANiAm and K. RAMAKRISHNAN, Rational approximants to 5-, 8-, and 7-fold two-dimensional tilings, Zeitschrift für Kristallographie 218 (2003), 590.

[27] H. UNKELBACH, Die kantensymmetrischen, gleichkantigen Polyeder, Deutsche Math. 5 (1940), 306316.

[28] M. VAN LEEUWEN, Computing Kazhdan-LustigVogan polynomials for split $E_{8}$, Nieuw Archief voor Wiskunde 9 (2008), 113-116. 